\title{
Brazil examines claim that research is losing out in science funding
}

São Luís, Brazil. Brazil's Ministry for Science and Technology has launched a review of the effectiveness of its expensive postgraduate scholarship funding programme, which some claim is distorting science funding.

According to Jose Galizia Tundisi, the new president of the National Council for Scientific and Technological Development (CNPq), the agency spends about 380 million reais (\$340 million) a year on scholarships - but only about 10 per cent of this figure on research grants. (CNPq is the ministry of science and technology's main funding agency.)

Tundisi told the annual meeting of the Brazilian Society for the Progress of Science (SBPC) in São Luís last month that he accepts that the country needs 100,000 active scientists. But he expressed concern about the quality of the research that they may be able to produce. "CNPq pays about 44,000 scholarships; I need to know what they are doing, and what sort of science the country is getting," he said.

The government is already supporting studies of scientific output, and a new programme - known as System for the Evaluation of Scholarships Abroad or SABE, the Portuguese word for 'knows' is now trying to measure how much Brazilian students and scientists have benefited from time spent abroad. According to Tundisi, the first results, based on interviews with one hundred returning scientists, show Brazil needs to take action to avoid duplication of research efforts.

The decision to pour resources into postgraduate education and training was made by the government of President José Sarney, which held office between 1985 and 1990. According to Crodowaldo Pavan, who headed CNPq during that period, the move was a deliberate effort to boost the country's scientific capabilities; he told the SBPC meeting that it is now up to Sarney's successors to find resources to keep Brazil's newly qualified scientists usefully occupied.

Pavan said politicians told him he should first obtain the money for scholarships in order to attract people into science, and that the money for research itself would then follow. "As a result, we have given more scholarships in three years than in the previous 31 years of CNPq's existence", says Pavan. But now the bill has arrived.

Lindolpho de Carvalho Dias, executive secretary at the ministry, says that efforts are now being made to correct these numbers. But he points to at least one advantage of the scheme, namely that "all good students are now receiving scholarships". Ricardo Bonalume Neto

\section{Synchrotron proposals spark high-energy debate in Japan}

Tokyo. A proposal by Tokyo University's Institute for Nuclear Study (INS) to build a powerful $50-\mathrm{GeV}$ proton synchrotron has split Japan's high-energy physics community. While some leading high-energy physicists support the proposed facility, others fear that it could undermine their plans to build the world's next giant linear collider.

The synchrotron would be a key facility at a planned new complex of three national research institutes in Tsukuba science city, north-east of Tokyo. The three institutes for high-energy physics, nuclear studies and synchrotron research - are expected to be set up in 1997 through reorganization of the National Laboratory for High Energy Physics (KEK) in Tsukuba, and the relocation of INS and its conversion to a national research institute, like KEK.

The proposed Japan Linear Collider (JLC) would consist of two linear accelerators (linacs), with a combined length of 25 $\mathrm{km}$, for colliding beams of electrons and positrons, each with energies of 150-250 $\mathrm{GeV}$ (see Nature 370, 169; 1994). Considerable research and development on the JLC has already been carried out at KEK.

The synchrotron proposal caught the high-energy physics community by surprise when it was announced by INS about two months ago, setting off a surge of protest by electronic mail. The idea was not entirely new, as INS has been discussing for about ten years its proposed Japan Hadron Project, which originally called for a $1-\mathrm{GeV}$ proton linear accelerator, but included the possibility of adding a high-energy synchrotron in a second phase (see Nature 353, 200; 1991). But the idea of starting with a powerful (and expensive) synchrotron is new.

Current plans for the synchrotron facility feature a $200-\mathrm{MeV}$ linac and a $3-\mathrm{GeV}$ booster ring, from which a small fraction of the beam will be tapped off to the main $50-\mathrm{GeV}$ ring. Sakue Yamada, director of INS, says the faculty’s cost is estimated at $¥ 75$ billion (US\$830 million). This is considerably more than the original plan, and supporters of the JLC fear it will make it more difficult for them to obtain the $¥ 200$ billion-plus needed for their own machine.

A meeting was held about a month ago between leading nuclear and high-energy physicists to try to calm the situation. Yamada says that the agitation among high-energy physicists "has cooled down", and hopes that supporters of the JLC accept that the synchrotron is not a threat to the linear collider, as the much larger budget for the collider would have to come from a different budget category. One JLC proponent rejects this argument. He says the general public is not aware of different budget categories, as it is "all the same tax" that pays for such facilities and the public "will probably ask why scientists need two such machines". But Yamada points out that, compared to highenergy physics, both nuclear and solid-state physics have received little government funding in recent years. He points out that the proposed synchrotron facility could be used by solid-state physicists and biologists as well as nuclear and high-energy physicists.

Apart from competition for funding within the physics community, a long-standing rivalry between the Ministry of Education, Science and Culture (Monbusho), which funds both INS and KEK, and the Science and Technology Agency (STA), has helped to launch the INS proposal. Ten years ago, the STA upset both Monbusho and the ministry's synchrotron researchers by deciding to build the world's largest electron synchrotron, the $8-\mathrm{GeV}$ SPring-8, which will be completed in a few years time in Nishi Harima, west of Osaka. In the process the STA "took some power away" from Monbusho, says one physicist at Tokyo University, and the education ministry is now seeking to regain its position with the INS proposal.

The STA is planning to build a very highpowered $1.5-\mathrm{GeV}$ proton linac as a neutron source and has the ultimate goal of using the proton beam to dispose of nuclear waste. Some efforts were made by researchers to coordinate this with the earlier - and less ambitious - Japan Hadron Project. But the talks did not progress far, and Monbusho and INS chose the more ambitious path of a $50-\mathrm{GeV}$ proton synchrotron in part to distinguish themselves from the STA project.

INS's proposal is to use the existing infrastructure of a $12-\mathrm{GeV}$ proton synchrotron at $\mathrm{KEK}$, which will be replaced by the $50-\mathrm{GeV}$ machine. The new synchrotron will be accommodated in a $1.4-\mathrm{km}$ circular tunnel within the main ring of KEK's TRISTAN positron-electron collider

The $3-\mathrm{GeV}$ booster will be used as a source of pulsed neutrons, muons and exotic nuclei for experiments to be carried out mainly by solid-state physicists. The $50-\mathrm{GeV}$ synchrotron will also produce high-intensity meson/neutrino beams, and will be used to extend intermediate energy nuclear and particle physics by, for example, carrying out very-fine-resolution experiments on hyper nuclei, hyperon-nucleon scattering, heavy ion reactions and kaon rare decays.

A special open meeting for high-energy physicists will be held next month to discuss the INS proposal and the JLC. According to Yamada, nuclear physicists will also participate and they hope to achieve "better understanding" between the two communities.

David Swinbanks 